\title{
Combining Ability and Heterosis for Grain Yield and its Component Traits in Rice (Oryza sativa L.)
}

\author{
Srikrishna LATHA ${ }^{1}$, Deepak SHARMA', Gulzar S. SANGHERA² \\ ${ }^{1}$ Indira Gandhi Kirshi Vishwavidyalaya, Department of Genetics and Plant Breeding, Raipur Chhattisgarh, India; deepakigkv@gmail.com \\ ${ }^{2}$ SKUAST-Kashmir, Mountain Research Centre for Field Crops, Khudwani, Anantnag, 192102, Kashmir, India; g_singh72@rediffmail.com
}

\begin{abstract}
The nature and magnitude of heterosis and combining ability was studied in $18 \mathrm{~F}_{1}$ rice hybrids involving three CMS lines and six testers using line $\times$ tester analysis. The analysis of variance for combining ability of all the traits showed that variances due to treatments, parents, hybrids were highly significant. The line 'CRMS 32A' and testers viz. 'Super rice-8', 'R 1099-2569-1-1' and 'Jitpiti' were identified as good general combiners. The significant differences between lines $\mathrm{x}$ testers interaction indicates that SCA attributed heavily in the expression of these traits and demonstrates the importance of dominance or non additive variances for all the traits. The hybrid 'CRMS 32A'/'R 1099-2569-1-1' and 'APMS 6A'/'Super rice-8' were promising for grain yield. The magnitude of relative heterosis, heterobeltiosis and standard heterosis were also estimated for different characters. A high degree of relative heterosis was observed for grain yield (20.4582.37\%) in the hybrids viz., 'CRMS 32A'/'Super rice-8,' APMS 6A'/'Super rice-8', 'APMS 6A'/'Jitpiti' and 'CRMS 32A'/'R 1099-2569-11'. While, a higher degree of: heterobeltiosis (13.60 -68.37\%) was observed for grain yield in the hybrids viz., 'CRMS 32A'/'Super rice-8', 'CRMS 32A'/'R 1099-2569-1-1', 'APMS 6A'/'Super rice-8' and 'APMS 6A'/'Jitpiti'. A high degree of standard heterosis was observed for grain yield in the hybrid 'CRMS 32A'/'R 1099-2569-1-1'. The hybrid 'CRMS 32A'/ 'R 1099-2569-1-1' recorded a high degree of relative heterosis (62.01\%), heterobeltiosis (57.35\%) and standard heterosis (15.05 and 25.51\% over check hybrids, 'Mahamaya' and 'Indirasona', respectively) that can be tested on yield trials for its further testing over locations.
\end{abstract}

Keywords: combining ability, heterosis, line $\times$ tester, rice

\section{Introduction}

Rice has been one of the world's most important food crops, feeding more than half of the world's population (Khush, 1997). In the Asia and Pacific region, rice is the main staple food and the most important source of employment and income for rural people (Hossain, 1998). The rice productivity has reached a plateau so it is thus imperative to find alternative means for increasing the yield potential of rice cultivars in a sustainable manner. Of the various approaches contemplated to break the existing yield barriers in rice, hybrid rice technology offers an opportunity to boost the yield of rice under fragile conditions as hybrid rice varieties have a yield advantage of $15-20 \%$ over the conventional high yielding varieties (Virmani et al., 1996). The success story of hybrid rice technology in China (Lin and Yuan, 1980) as leading producer of hybrid rice in the world (Swaminathan, 2006) and some other countries along with India has been witnessed as an important and readily adoptable genetic option to increase the rice production and offers a viable solution to meet the ever increasing food challenge in different countries (Rai, 2009; Sanghera and Wani, 2008; Virmani et al., 2003).

Breeding strategies for developing hybrids with high yield potential and better grain quality require the expect- ed level of heterosis and combining ability. Knowledge of Combining ability and heterosis helps in the selection of appropriate parents for a hybridization programme for evolving elite segregants with high grain yield in the segregating generations.

In order to exploit maximum heterosis using the CMS system in a hybrid programme, one must know the combining ability of different male sterile and restorer lines. The knowledge of combining ability allows the assessment of nicking ability among genotypes and understanding of the nature and magnitude of gene actions involved. Its role is important to decide parents, crosses and appropriate breeding procedures to be followed to select desirable segregants (Salgotra et al., 2009). The general combining ability (GCA) identifies superior parental genotypes while specific combining ability (SCA) helps in identification of good hybrid combinations which may ultimately lead to the development of hybrids (Saleem et al., 2008). Line $\mathrm{x}$ Tester (Kempthorne, 1957) analysis is one of the most powerful tools for estimating the GCA of parents and selection of desirable parents and crosses with high SCA for the exploitation of heterosis (Rashid et al., 2007; Sarkar et al., 2002; Sanghera and Hussain, 2012a, b; Tiwari et al., 2011 , ). The present investigation was conducted to assess the combining ability of male sterile and identified restor- 
er lines for the exploitation of maximum heterosis in $F_{1}$ hybrids for yield and yield contributing traits.

\section{Materials and methods}

The present study entitled was conducted at the University Research cum Instructional Farm, Department of Genetics and Plant breeding, College of Agriculture, Indira Gandhi Kirshi Vishwavidyalaya, Raipur (Chhattisgarh) during wet season 2011. It is situated at $21^{\circ} 16 \mathrm{~N}$ Latitude and $81^{\circ} 36^{\prime} \mathrm{E}$ longitude at an altitude of 289.60 meters above mean sea level. It comes under sub-humid region receiving an average rainfall of $1400 \mathrm{~mm}$ annually, of which about 92 percent is received during rainy season between June to September and remaining 8 percent during winter season between October to March. The experimental material comprised of three CMS lines ('APMS 6A', 'CRMS 32A' and 'PUSA 6A') used as females and six testers ('SR-6-SW-8-1', 'R 1099-2569-1-1', 'R-1557-13061-568-1', 'Super rice-7', 'Jitpiti' and 'Super rice-8') used as males and resulting $18 \mathrm{~F}_{1}$ hybrids obtained from line $\times$ tester mating design. The twenty one days old seedlings of $18 \mathrm{~F}_{1}$ hybrids along with their parents were transplanted in the main field during 2011 at Research farm, IGKV, Raipur, Chhattisgarh. The experiment was conducted as randomized complete block design with two replications with inter-row and intra-row spacing of $20 \mathrm{~cm}$ having a plot size of $5 \mathrm{~m} \times 1 \mathrm{~m}$. All recommended agronomical practices were followed to raise the ideal crop stand. Observations were recorded on ten agro-morphological characters such as days to 50 per cent flowering, plant height (cm), number of tillers per plant, number of productive tillers per plant, panicle length $(\mathrm{cm})$, pollen fertility $(\%)$, spikelet fertility (\%), biological yield per plant (g) and grain yield per plant $(\mathrm{g})$ and 1000 - grain weight $(\mathrm{g})$. The mean data were recorded on five randomly selected plants from parents and $\mathrm{F}_{1}$ 's from each replication. Heterosis was estimated from mean values according to the Fehr (1987). The significance of different types of heterosis was carried out by adopting ' $t$ test' as suggested by Nadarajan and Gunasekaran (2005) as given below:

$$
\begin{aligned}
& t(\text { relative heterosis })=\frac{\overline{F_{i j}}-\overline{\mathrm{MP}_{i j}}}{\overline{\mathrm{SE}}} \times 100 \\
& \mathrm{t}(\text { heterobeltosis })=\frac{\overline{\mathrm{F}_{\mathrm{ij}}}-\overline{\mathrm{BP}_{\mathrm{ij}}}}{\overline{\mathrm{SE}}} \times 100 \\
& \mathrm{t}(\text { Standard heterosis })=\frac{\overline{\mathrm{F}_{\mathrm{ij}}}-\overline{\mathrm{CV}}}{\overline{\mathrm{SE}}} \times 100
\end{aligned}
$$

Where: Fij-F1 cross of $\mathrm{i}$ and $\mathrm{j}$ parent, MP- mean mid parental performance same cross, BP-Mean performance of better parent and CV-mean performance of commercial variety

However, combining ability analysis was done using line $\times$ tester method (Kempthorne, 1957). The variances for general combining ability (GCA) and specific combining ability (SCA) were tested against their respective error variances derived from ANOVA reduced to mean level. Significance test for GCA and SCA effects were performed using t-test.

Significance of GCA effects of lines is tested as:

$$
\mathrm{t}=\frac{\mathrm{g}_{\mathrm{i}}}{\mathrm{SE}\left(\mathrm{g}_{\mathrm{i}}\right)}
$$

Significance of GCA effects of testers is tested as:

$$
\mathrm{t}=\frac{\mathrm{g}_{\mathrm{j}}}{S E\left(\mathrm{~g}_{\mathrm{j}}\right)}
$$

Significance of SCA effects of hybrids is tested as:

$$
t=\frac{S_{i j}}{S E\left(S_{i j}\right)}
$$

\section{Results and discussion}

\section{General and specific combining ability}

The analysis of variance for combining ability of all the traits under study is presented in the Tab. 1, which showed that variances due to treatments (parents+ crosses), parents, hybrids were highly significant for all the characters. The variance due to parent vs. hybrids was also found highly significant for almost all the characters except for test weight. The variance due to lines was found non-significant for panicle length. The variance due to testers was found significant all the characters except tillers per plant, productive tillers per plant and panicle length. The significant difference between parents and crosses for most of traits indicates that they are suitable for genetic studies. Further, the significant mean squares of parent vs. crosses in most of studied traits revealed scope of heterosis for these traits. These results coincide with the findings of (Jayasudha and Sharma, 2009; Rahimi et al., 2010), where significant difference among parents vs. crosses were also found. The significant differences between lines $x$ testers interaction indicates that SCA attributed heavily in the expression of these traits and demonstrates the importance of dominance or non additive variances for all the traits. The significant mean squares of lines and testers also revealed the prevalence of additive variances for the traits studied. Occurrence of both additive and non additive gene effects for yield and important yield component traits in rice has been reported in earlier studies (Faiz et al., 2006; Rahimi et al., 2010; Sanghera and Hussain, 2012a).

The results revealed that none of the parents showed significant GCA effects simultaneously in the desired direction for all the traits studied (Tab. 2a). However, to determine the appropriate parent for subsequent hybrid rice development, variation in GCA effects was estimated among lines and testers for all traits. Negative GCA effects 
Tab. 1. Analysis of variance for line $\times$ tester and combining ability for grain yield and component traits in rice

\begin{tabular}{cccccccccccc}
\hline Source & d.f & $\begin{array}{c}\text { Days } \\
\text { to } 50 \% \\
\text { lowering }\end{array}$ & $\begin{array}{c}\text { Plant } \\
\text { height }\end{array}$ & $\begin{array}{c}\text { Tillers } \\
\text { per } \\
\text { plant }\end{array}$ & $\begin{array}{c}\text { Productive } \\
\text { tillers per } \\
\text { plant }\end{array}$ & $\begin{array}{c}\text { Panicle } \\
\text { length }\end{array}$ & $\begin{array}{c}\text { Pollen } \\
\text { fetility } \\
(\%)\end{array}$ & $\begin{array}{c}\text { Spikelet } \\
\text { fetility } \\
(\%)\end{array}$ & $\begin{array}{c}1000 \\
\text { grain } \\
\text { weight }\end{array}$ & $\begin{array}{c}\text { Biological } \\
\text { yield }\end{array}$ & $\begin{array}{c}\text { Grain } \\
\text { yield }\end{array}$ \\
\hline Replications & 1 & 2.77 & 7.38 & 2.41 & 0.14 & 0.18 & 13.08 & 0.17 & 2.35 & 1.35 & 1.04 \\
\hline Parents & 8 & $45.27^{* *}$ & $125.55^{* *}$ & $7.80^{* *}$ & $6.75^{* *}$ & $6.36^{* *}$ & $3729.8^{* *}$ & $51.33^{* *}$ & $11.47^{* *}$ & $267.07^{* *}$ & $53.89^{* *}$ \\
\hline Hybrids & 17 & $39.00^{* *}$ & $52.93^{* *}$ & 0.06 & 1.99 & 1.69 & $1563.83^{* *}$ & $1407.11^{* *}$ & $8.94^{* *}$ & $899.09^{* *}$ & $228.02^{* *}$ \\
\hline Parents vs Hybrids & 1 & $201.06^{* *}$ & $28.87^{* *}$ & 1.92 & 0.20 & $45.60^{* *}$ & $566.86^{* *}$ & $14826.5^{* *}$ & 1.29 & $743.03^{* *}$ & $79.67^{* *}$ \\
\hline Lines & 2 & $68.08^{* *}$ & $15.42^{* *}$ & $6.23^{* *}$ & $6.24^{* *}$ & 2.44 & $239.39^{* *}$ & $242.22^{* *}$ & $24.24^{* *}$ & $2690.49^{* *}$ & $218.44^{* *}$ \\
\hline Testers & 5 & $79.73^{* *}$ & $92.51^{* *}$ & 1.06 & 0.86 & 0.42 & $4019.30^{* *}$ & $3625.39^{* *}$ & $13.36^{* *}$ & $593.31^{* *}$ & $422.63^{* *}$ \\
Line $\times$ Tester & 10 & $12.81^{* *}$ & $40.65^{*}$ & 1.49 & 1.71 & 2.18 & $600.98^{* *}$ & $530.95^{* *}$ & $3.66^{* *}$ & $693.70^{* *}$ & $132.63^{* *}$ \\
\hline Error & 17 & 0.71 & 12.23 & 0.43 & 0.48 & 1.06 & 1.97 & 2.37 & 0.85 & 4.97 & 1.95 \\
\hline $\begin{array}{l}\text { Variance of GCA } \\
\text { Variance of SCA }\end{array}$ & 6.78 & 1.48 & 0.22 & 0.27 & -0.02 & 203.09 & 18.57 & 1.83 & 143.61 & 20.87 \\
\hline $\begin{array}{c}\text { Variance of } \\
\text { GCA/ Variance } \\
\text { of SCA }\end{array}$ & 6.05 & 14.20 & 0.53 & 0.61 & -0.56 & 299.50 & 264.29 & 1.40 & 344.36 & 65.34 \\
\hline
\end{tabular}

${ }^{*}=$ significant of $\mathrm{p}=0.05$ level, ${ }^{* *}=$ significant of $\mathrm{p}=0.01$ level

Tab. 2a. Estimtes of general combining ability (GCA) effects for different characters in rice

\begin{tabular}{|c|c|c|c|c|c|c|c|c|c|c|}
\hline Parents & $\begin{array}{c}\text { Days } \\
\text { to } 50 \% \\
\text { flowering }\end{array}$ & $\begin{array}{l}\text { Plant } \\
\text { height } \\
(\mathrm{cm})\end{array}$ & $\begin{array}{c}\text { Tillers } \\
\text { per plant }\end{array}$ & $\begin{array}{c}\text { Productive } \\
\text { tillers per } \\
\text { plant }\end{array}$ & $\begin{array}{c}\text { Panicle } \\
\text { length } \\
(\mathrm{cm})\end{array}$ & $\begin{array}{c}\text { Pollen } \\
\text { fetility } \\
(\%)\end{array}$ & $\begin{array}{c}\text { Spikelet } \\
\text { fetility } \\
(\%)\end{array}$ & $\begin{array}{l}1000 \text { grain } \\
\text { weight }(\mathrm{g})\end{array}$ & $\begin{array}{l}\text { Biological } \\
\text { yield }(\mathrm{g})\end{array}$ & $\begin{array}{c}\text { Grain } \\
\text { yield }(\mathrm{g})\end{array}$ \\
\hline \multicolumn{11}{|c|}{ Lines } \\
\hline 'APMS 6A' & $-2.66^{* *}$ & 1.11 & 0.21 & 0.15 & -0.29 & $-3.48^{* *}$ & $-3.37^{* *}$ & $-1.44^{* *}$ & $2.71^{* *}$ & 0.30 \\
\hline 'CRMS 32A' & $1.91^{* *}$ & 0.02 & $0.58^{* *}$ & $0.62^{* *}$ & 0.52 & $5.03^{* *}$ & $5.09^{* *}$ & 0.04 & $13.42^{* *}$ & $4.10^{* *}$ \\
\hline 'PUSA 6A' & $0.75^{* *}$ & -1.14 & $-0.80^{* *}$ & $-0.78^{* *}$ & -0.22 & $-1.55^{* *}$ & $-1.72^{* *}$ & $1.39^{* *}$ & $-16.14^{* *}$ & $-4.41^{* *}$ \\
\hline SE(Lines) & 0.24 & 0.86 & 0.16 & 0.19 & 0.26 & 0.37 & 0.39 & 0.24 & 0.58 & 0.35 \\
\hline \multicolumn{11}{|c|}{ Testers } \\
\hline 'Super rice-8' & $1.50^{* *}$ & $4.31^{* *}$ & 0.32 & 0.31 & 0.37 & $19.33^{* *}$ & $17.76^{* *}$ & $2.12^{* *}$ & $5.37^{* *}$ & $4.33^{* *}$ \\
\hline 'R 1099-2569-1-1' & $4.66^{* *}$ & 1.75 & -0.26 & -0.18 & 0.11 & $14.88^{* *}$ & $14.79^{* *}$ & 0.22 & $6.72^{* *}$ & $7.96^{* *}$ \\
\hline 'R1557-1306-1-568-1' & $3.16^{* *}$ & 1.01 & -0.46 & -0.55 & -0.12 & $-45.51^{* *}$ & $-43.36^{* *}$ & $-2.06^{* *}$ & $5.12^{* *}$ & $-13.13^{* *}$ \\
\hline 'SR-6-SW-8-1' & $-4.16^{* *}$ & $-2.68^{*}$ & $0.68^{*}$ & 0.52 & -0.35 & $-16.84^{* *}$ & $-15.81^{* *}$ & $-1.32^{* *}$ & $-4.62^{* *}$ & $-7.33^{* *}$ \\
\hline 'Jitpiti' & $-3.50^{* *}$ & 2.11 & -0.12 & -0.05 & 0.17 & $15.28^{* *}$ & $14.19^{* *}$ & 0.40 & $5.79^{* *}$ & $6.34^{* *}$ \\
\hline 'Super rice-7' & $-1.66^{* *}$ & $-6.53^{* *}$ & -0.14 & -0.03 & -0.17 & $12.85^{* *}$ & $12.43^{* *}$ & 0.63 & $-18.41^{* *}$ & $1.83^{* *}$ \\
\hline SE (Testers) & 0.34 & 1.21 & 0.24 & 0.27 & 0.37 & 0.53 & 0.55 & 0.34 & 0.82 & 0.49 \\
\hline
\end{tabular}

${ }^{*}=$ significant of $\mathrm{p}=0.05$ level, ${ }^{* *}=$ significant of $\mathrm{p}=0.01$ level

were desirable for days to 50 percent flowering and plant height while in other traits positive GCA effects were desirable. Character wise estimation of GCA effects of lines (Tab. 2a) revealed the line 'CRMS 32A' to be a good combiner of grain yield and several contributing characters viz., tillers per plant, productive tillers per plant, panicle length, pollen fertility percentage and spikelet fertility percentage. 'APMS 6A' to be a good combiner for days to 50 percent flowering and 'PUSA $6 \mathrm{~A}$ ' is a good combiner for thousand grain weight. GCA effects of testers also revealed that 'Super rice- 8 ' is good combiner for characters $v i z$., pollen fertility percentage, spikelet fertility percentage, biological yield, grain yield and 1000 grain weight. ' $R$ 1099-2569-1-1' and 'Jitpiti' is a good combiner for pollen fertility percentage, spikelet fertility percentage, biological yield, grain yield. These results are in complete agreement with earlier findings of Anand et al. (1999), Vanaja et al. (2003). Investigation of GCA effects revealed that among lines and testers were good general combiners for grain yield and other traits. Hence, these good general combiners of males and females may be extensively used in future for hybrid rice breeding programme.

Based on the estimates of SCA effects none of the cross combinations exhibited significant and desirable SCA effect for all the parameters simultaneously (Tab. 2b) indicating that no specific combination was desirable for all traits. These results are in complete agreement with earlier findings (Sanghera and Hussain, 2012b; Tiwari et al., 2011). Yield is ultimate goal of a rice breeding and hybrid development programme. Character wise estimation of 
Tab. 2b. Estmates of specific combining ability (SCA) effects for different characters in rice

\begin{tabular}{|c|c|c|c|c|c|c|c|c|c|c|}
\hline Hybrids & $\begin{array}{c}\text { Days } \\
\text { to } 50 \% \\
\text { flowering }\end{array}$ & $\begin{array}{l}\text { Plant } \\
\text { height } \\
(\mathrm{cm})\end{array}$ & $\begin{array}{c}\text { Tillers } \\
\text { per plant }\end{array}$ & $\begin{array}{c}\text { Productive } \\
\text { tillers per } \\
\text { plant }\end{array}$ & $\begin{array}{l}\text { Panicle } \\
\text { length } \\
(\mathrm{cm})\end{array}$ & $\begin{array}{c}\text { Pollen } \\
\text { fertility } \\
(\%)\end{array}$ & $\begin{array}{c}\text { Spikelet } \\
\text { fertility } \\
(\%)\end{array}$ & $\begin{array}{c}1000 \\
\text { grain } \\
\text { wt }(\mathrm{g}) \\
\end{array}$ & $\begin{array}{c}\text { Biological } \\
\text { yield }(\mathrm{g})\end{array}$ & $\begin{array}{c}\text { Grain } \\
\text { yield }(\mathrm{g})\end{array}$ \\
\hline \multicolumn{11}{|c|}{ 'APMS 6A' } \\
\hline 'Super rice-8' & $-2.0^{* *}$ & $6.12^{*}$ & 0.03 & 0.20 & 0.49 & $19.44^{* *}$ & $15.51^{* *}$ & $0.83^{*}$ & $18.83^{* *}$ & $11.10^{* *}$ \\
\hline 'R1099-2569-1-1' & $2.33^{* *}$ & 4.38 & 0.12 & 0.10 & 0.77 & $5.19^{* *}$ & $5.41^{* *}$ & $0.93^{*}$ & $14.48^{* * *}$ & -1.43 \\
\hline 'R1557-1306-1-558-1' & -0.67 & -4.38 & $1.02^{*}$ & 0.87 & 0.10 & 1.74 & $2.14^{*}$ & $-1.49^{*}$ & $-11.72^{*}$ & -0.56 \\
\hline 'SR-6-SW-8-1' & 0.66 & -5.08 & -0.93 & $-1.10^{*}$ & -0.11 & $-29.36^{* *}$ & $-27.33^{* *}$ & $-0.86^{*}$ & -2.17 & 0.64 \\
\hline 'Jitpiti' & $-2.0^{* *}$ & -0.28 & -0.12 & -0.03 & 0.51 & $10.66^{* *}$ & $12.01^{* *}$ & $-1.71^{*}$ & $9.22^{* *}$ & $5.15^{* *}$ \\
\hline 'Super rice -7 ’’ & $1.6^{*}$ & -0.73 & -0.15 & -0.10 & $-1.73^{*}$ & $-7.67^{* *}$ & $-7.47^{* *}$ & $0.56^{*}$ & $-15.64^{* *}$ & $-8.48^{* *}$ \\
\hline \multicolumn{11}{|c|}{ 'CRMS 32A' } \\
\hline 'Super rice-8' & $-2.58^{* *}$ & -0.89 & 0.46 & 0.53 & -0.46 & -0.98 & $2.38^{*}$ & $1.44^{*}$ & $7.96^{* *}$ & $-27.30^{*}$ \\
\hline 'R1099-2569-1-1' & 0.25 & 1.07 & 0.65 & 0.63 & 0.36 & $21.95^{* *}$ & $19.13^{* *}$ & $1.09^{*}$ & $19.36^{* *}$ & $11.17^{*}$ \\
\hline 'R1557-1306-1-558-1' & 0.25 & 0.41 & $-0.95^{*}$ & -0.80 & 0.04 & $-55.67^{* *}$ & $-8.63^{* *}$ & $0.97^{*}$ & $-4.24^{*}$ & $-6.16^{* *}$ \\
\hline 'SR-6-SW-8-1' & $3.66^{* *}$ & 0.47 & -0.70 & -0.77 & 0.29 & $7.46^{* *}$ & $6.85^{*}$ & $-1.96^{* *}$ & $-18.69^{* *}$ & $-3.36^{* *}$ \\
\hline 'Jitpiti' & $7.0^{* *}$ & -1.13 & 0.21 & 0.10 & -1.51 & $-4.61^{* *}$ & $-5.16^{* *}$ & $1.25^{*}$ & $7.70^{* *}$ & $-2.75^{*}$ \\
\hline 'Super rice -7 ’’ & $5.16^{* *}$ & 0.21 & 0.33 & 0.28 & 1.25 & $-3.00^{* *}$ & $-4.51^{* *}$ & $-0.23^{*}$ & $-3.49^{* *}$ & $-2.38^{*}$ \\
\hline \multicolumn{11}{|c|}{ 'PUSA 6A' } \\
\hline 'Super rice -8 ' & $4.59^{* *}$ & $-5.22^{*}$ & -0.30 & -0.75 & -0.03 & $-18.44^{* *}$ & $-14.85^{* *}$ & $-0.36^{*}$ & $-15.16^{* *}$ & $-8.25^{* *}$ \\
\hline 'R1099-2569-1-1' & $-2.58^{*}$ & $-5.46^{*}$ & -0.76 & -0.75 & -1.13 & $-15.78^{* *}$ & $-14.28^{* *}$ & $-1.36^{*}$ & $-23.86^{* *}$ & $-9.72^{* *}$ \\
\hline 'R1557-1306-1-558-1' & -1.07 & 4.04 & -0.06 & -0.08 & -0.14 & $-38.74^{* *}$ & $6.51^{* *}$ & $0.52^{*}$ & $15.94^{* *}$ & $6.75^{* *}$ \\
\hline 'SR-6-SW-8-1' & 0.25 & 4.74 & $1.59^{*}$ & $1.85^{* *}$ & -0.19 & $6.59^{* *}$ & $8.58^{* *}$ & $0.83^{*}$ & $15.36^{* *}$ & $2.75^{*}$ \\
\hline 'Jitpiti' & 0.35 & 1.54 & -0.10 & -0.08 & 0.98 & $-6.02^{* *}$ & $-6.79^{* *}$ & $0.45^{*}$ & $-16.92^{* *}$ & $-2.43^{*}$ \\
\hline 'Super rice -7’ & $-2.24^{* *}$ & 0.54 & -0.18 & -0.20 & 0.49 & $10.7^{* *}$ & $12.06^{* *}$ & $-0.33^{*}$ & $9.14^{* *}$ & $4.74^{* *}$ \\
\hline $\mathrm{SE}_{\mathrm{m \pm}}$ & 0.59 & 2.47 & 0.45 & 0.48 & 0.72 & 0.98 & 1.08 & 0.64 & 1.57 & 0.98 \\
\hline
\end{tabular}

${ }^{*}=$ significant of $\mathrm{p}=0.05$ level; ${ }^{* *}=$ significant of $\mathrm{p}=0.01$ level

Tab. 3. Percent relative heterosis, heterobeltiosis and standard heterosis for different characters in rice

\begin{tabular}{|c|c|c|c|c|c|c|c|c|}
\hline \multirow[t]{2}{*}{ Hybrids } & \multicolumn{4}{|c|}{ Days to $50 \%$ flowering } & \multicolumn{4}{|c|}{ Plant height $(\mathrm{cm})$} \\
\hline & MP & $\mathrm{BP}$ & 'Mahamaya' & 'Indirasona' & MP & $\mathrm{BP}$ & 'Mahamaya' & 'Indirasona' \\
\hline \multicolumn{9}{|c|}{ 'APMS 6A' } \\
\hline 'Super rice-8' & $-4.97^{* *}$ & $-9.47^{* *}$ & 1.20 & $-7.14^{* *}$ & $5.96^{*}$ & 4.45 & $9.02^{* *}$ & $6.31^{*}$ \\
\hline 'R1099-2569-1-1' & $2.03^{*}$ & $-4.00^{* *}$ & $10.18^{* *}$ & 1.10 & 0.86 & 0.70 & 5.10 & 2.49 \\
\hline 'R1557-1306-1-568-1' & $-4.11^{* *}$ & $-10.71^{* *}$ & $4.79^{* *}$ & $-3.85^{* *}$ & -2.61 & $-7.59^{* *}$ & -3.55 & $-5.95^{*}$ \\
\hline 'SR-6-SW-8-1' & $-6.05^{* *}$ & $-8.43^{* *}$ & $-2.40^{*}$ & $-10.44^{* *}$ & $-10.43^{* *}$ & $-11.43^{* *}$ & $-7.56^{*}$ & $-9.86^{* *}$ \\
\hline 'Jitpiti' & $-10.51^{* *}$ & $-14.67^{* *}$ & $-4.79^{* *}$ & $-12.64^{* *}$ & -1.56 & -3.05 & 1.18 & -1.33 \\
\hline 'Super rice-7' & $-4.76^{* *}$ & $-9.57^{* *}$ & 1.80 & $-6.59^{* *}$ & $-6.22 *$ & $-10.99^{* *}$ & $-7.10^{*}$ & $-9.41^{* *}$ \\
\hline \multicolumn{9}{|c|}{ 'CRMS 32A' } \\
\hline 'Super rice-8' & $-5.26^{* *}$ & $-5.35^{* *}$ & $5.99^{* *}$ & $-2.75^{* *}$ & $9.64^{* *}$ & 0.24 & 1.64 & -0.89 \\
\hline 'R1099-2569-1-1' & -0.18 & -1.39 & $13.17^{* *}$ & $3.85^{* *}$ & $7.52^{* *}$ & -2.83 & 1.09 & -1.42 \\
\hline 'R1557-1306-1-568-1' & $-2.87^{* *}$ & $-5.10^{* *}$ & $11.38^{* *}$ & $2.20^{*}$ & $12.35^{* *}$ & $6.55^{*}$ & -0.18 & -2.66 \\
\hline 'SR-6-SW-8-1' & $-7.40^{* *}$ & $-9.63^{* *}$ & 1.20 & $-7.14^{* *}$ & 3.68 & -5.48 & -3.55 & $-5.95 *$ \\
\hline 'Jitpiti' & $-5.18^{* *}$ & $-5.35^{* *}$ & $5.99^{* *}$ & $-2.75^{* *}$ & $7.29^{*}$ & -1.83 & -0.64 & -3.11 \\
\hline 'Super rice-7' & $-5.60^{* *}$ & $-5.85^{* *}$ & $5.99^{* *}$ & $-2.75^{* *}$ & 4.37 & -1.05 & $-7.24^{*}$ & $-9.55^{* *}$ \\
\hline \multicolumn{9}{|c|}{ 'PUSA 6A' } \\
\hline 'Super rice-8' & $6.28^{* *}$ & 1.25 & $13.17^{* *}$ & $3.85^{* *}$ & 1.37 & -4.70 & -3.37 & $-5.77^{*}$ \\
\hline 'R1099-2569-1-1' & 0.37 & $-5.57^{* *}$ & $8.38^{* *}$ & -0.55 & -2.65 & $-9.57^{* *}$ & $-5.92 *$ & $-8.26^{* *}$ \\
\hline 'R1557-1306-1-568-1' & 0.82 & $-6.12^{* *}$ & $10.18^{* *}$ & 1.10 & $11.52^{* *}$ & $8.88^{* *}$ & 2.00 & -0.53 \\
\hline 'SR-6-SW-8-1' & $-2.59^{* *}$ & $-5.06^{* *}$ & 1.20 & $-7.14^{* *}$ & 3.79 & -2.71 & -0.73 & -3.20 \\
\hline 'Jitpiti' & $-4.88^{* *}$ & $-9.31^{* *}$ & 1.20 & $-7.14^{* *}$ & $5.77^{*}$ & -0.48 & 0.73 & -1.78 \\
\hline 'Super rice-7’' & $-5.32^{* *}$ & $-10.11^{* *}$ & 1.20 & $-7.14^{* *}$ & 0.53 & -1.88 & $-8.01^{* *}$ & $-10.30^{* *}$ \\
\hline
\end{tabular}


Tab. 3. Percent relative heterosis, heterobeltiosis and standard heterosis for different characters in rice (Continuous)

\begin{tabular}{|c|c|c|c|c|c|c|c|c|}
\hline \multirow[t]{2}{*}{ Hybrids } & \multicolumn{4}{|c|}{ Tillers per plant } & \multicolumn{4}{|c|}{ Productive tillers per plant } \\
\hline & $\mathrm{MP}$ & $\mathrm{BP}$ & 'Mahamaya' & 'Indirasona' & MP & $\mathrm{BP}$ & 'Mahamaya' & 'Indirasona' \\
\hline \multicolumn{9}{|c|}{ 'APMS 6A' } \\
\hline 'Super rice-8' & -8.33 & $-26.85^{* *}$ & 6.76 & $-32.48^{* *}$ & -5.02 & $-23.30^{* *}$ & 12.86 & $-28.83^{* *}$ \\
\hline 'R1099-2569-1-1' & $-17.48^{* *}$ & $-31.48^{* *}$ & 0.00 & $-36.75^{* *}$ & $-15.95^{*}$ & $-29.13^{* *}$ & 4.29 & $-34.23^{* *}$ \\
\hline 'R1557-1306-1-568-1' & 14.61 & $-25.00^{* *}$ & 9.46 & $-30.77^{* *}$ & 12.94 & $-25.24^{* *}$ & 10.00 & $-30.63^{* *}$ \\
\hline 'SR-6-SW-8-1' & $-20.76^{* *}$ & $-32.13^{* *}$ & -0.95 & $-37.35^{* *}$ & $-23.81^{* *}$ & $-33.69^{* *}$ & -2.43 & $-38.47^{* *}$ \\
\hline 'Jitpiti' & $-14.12^{*}$ & $-32.41^{* *}$ & -1.35 & $-37.61^{* *}$ & -11.52 & $-29.13^{* *}$ & 4.29 & $-34.23^{* *}$ \\
\hline 'Super rice-7’' & $-21.62^{* *}$ & $-32.87^{* *}$ & -2.03 & $-38.03^{* *}$ & $-19.15^{* *}$ & $-29.61^{* *}$ & 3.57 & $-34.68^{* *}$ \\
\hline \multicolumn{9}{|c|}{ 'CRMS 32A' } \\
\hline 'Super rice-8' & $20.54^{* *}$ & 8.75 & $17.57^{*}$ & $-25.64^{* *}$ & $23.10^{*}$ & 11.54 & $24.29^{*}$ & $-21.62^{* *}$ \\
\hline 'R1099-2569-1-1' & 9.68 & 3.75 & 12.16 & $-29.06^{* *}$ & 11.63 & 6.41 & 18.57 & $-25.23^{* *}$ \\
\hline 'R1557-1306-1-568-1' & 14.69 & $-18.75^{*}$ & -12.16 & $-44.44^{* *}$ & 16.75 & -16.67 & -7.14 & $-41.44^{* *}$ \\
\hline 'SR-6-SW-8-1' & 0.64 & -1.25 & 6.76 & $-32.48^{* *}$ & -1.49 & -2.56 & 8.57 & $-31.53^{* *}$ \\
\hline 'Jitpiti’ & 12.68 & 0.00 & 8.11 & $-31.62^{* *}$ & 12.86 & 1.28 & 12.86 & $-28.83^{* *}$ \\
\hline 'Super rice-7' & 3.18 & 1.25 & 9.46 & $-30.77^{* *}$ & 4.96 & 3.85 & 15.71 & $-27.03^{* *}$ \\
\hline \multicolumn{9}{|c|}{ 'PUSA 6A' } \\
\hline 'Super rice-8' & -12.02 & $-20.63^{*}$ & -14.19 & $-45.73^{* *}$ & -13.26 & $-20.00^{*}$ & -14.29 & $-45.95^{* *}$ \\
\hline 'R1099-2569-1-1' & $-27.32^{* *}$ & $-31.25^{* *}$ & $-25.68^{* *}$ & $-52.99^{* *}$ & $-24.50^{* *}$ & $-26.67^{* *}$ & $-21.43^{*}$ & $-50.45^{* *}$ \\
\hline 'R1557-1306-1-568-1' & 5.87 & $-25.00^{* *}$ & $-18.92^{*}$ & $-48.72^{* *}$ & 7.06 & $-22.67^{*}$ & -17.14 & $-47.75^{* *}$ \\
\hline 'SR-6-SW-8-1' & 12.10 & 10.00 & $18.92^{*}$ & $-24.79^{* *}$ & 16.33 & 15.33 & $25.71^{*}$ & $-20.72^{* *}$ \\
\hline 'Jitpiti' & -11.27 & $-21.25^{*}$ & -14.86 & $-46.15^{* *}$ & -8.03 & -16.00 & -10.00 & $-43.24^{* *}$ \\
\hline 'Super rice-7' & $-21.02^{* *}$ & $-22.50^{* *}$ & -16.22 & $-47.01^{* *}$ & $-18.07^{*}$ & -18.80 & -11.43 & $-44.14^{* *}$ \\
\hline & \multicolumn{4}{|c|}{ Panicle length $(\mathrm{cm})$} & \multicolumn{4}{|c|}{ Pollen fertility (\%) } \\
\hline & $\mathrm{MP}$ & $\mathrm{BP}$ & 'Mahamaya' & 'Indirasona' & MP & $\mathrm{BP}$ & 'Mahamaya' & 'Indirasona' \\
\hline \multicolumn{9}{|c|}{ 'APMS 6A' } \\
\hline 'Super rice-8' & $11.90^{* *}$ & $9.86^{*}$ & $21.47^{* *}$ & 6.74 & $97.75^{* *}$ & -1.11 & $-3.26^{*}$ & $-6.47^{* *}$ \\
\hline 'R1099-2569-1-1' & 3.99 & -1.35 & $21.55^{* *}$ & 6.82 & $50.20^{* *}$ & $-24.89^{* *}$ & $-24.25^{* *}$ & $-26.77^{* *}$ \\
\hline 'R1557-1306-1-568-1' & $10.67^{* *}$ & 6.39 & $17.63^{* *}$ & 3.37 & $-91.88^{* *}$ & $-95.94^{* *}$ & $-96.17^{* *}$ & $-96.30^{* *}$ \\
\hline 'SR-6-SW-8-1' & -0.56 & -5.31 & $15.75^{* *}$ & 1.72 & $-97.70^{* *}$ & $-98.85^{* *}$ & $-98.93^{* *}$ & $-98.96^{* *}$ \\
\hline 'Jitpiti' & $9.35^{* *}$ & $9.20^{*}$ & $20.73^{* *}$ & 6.10 & $68.61^{* *}$ & $-15.68^{* *}$ & $-17.72^{* *}$ & $-20.45^{* *}$ \\
\hline 'Super rice-7' & -0.96 & -0.96 & $9.50^{*}$ & -3.77 & $19.33^{* *}$ & $-40.32^{* *}$ & $-41.06^{* *}$ & $-43.02^{* *}$ \\
\hline \multicolumn{9}{|c|}{ 'CRMS 32A' } \\
\hline 'Super rice-8' & $16.31^{* *}$ & $13.49^{* *}$ & $20.91^{* *}$ & 6.25 & $70.36^{* *}$ & $-14.80^{* *}$ & $-16.66^{* *}$ & $-19.42^{* *}$ \\
\hline 'R1099-2569-1-1' & $9.82^{* *}$ & 0.09 & $23.32^{* *}$ & $8.37^{*}$ & $80.99^{* *}$ & $-9.49^{* *}$ & $-8.72^{* *}$ & $-11.75^{* *}$ \\
\hline 'R1557-1306-1-568-1' & $18.88^{* *}$ & $18.50^{* *}$ & $20.91^{* *}$ & 6.25 & $-97.95^{* *}$ & $-98.97^{* *}$ & $-99.03^{* *}$ & $-99.06^{* *}$ \\
\hline 'SR-6-SW-8-1' & $8.25^{*}$ & -0.99 & $21.03^{* *}$ & 6.36 & $11.45^{* *}$ & $-44.26^{* *}$ & $-47.89^{* *}$ & $-49.62^{* *}$ \\
\hline 'Jitpiti' & $9.21^{*}$ & 4.81 & $15.56^{* *}$ & 1.55 & $53.02^{* *}$ & $-23.47^{* *}$ & $-25.32^{* *}$ & $-27.80^{* *}$ \\
\hline 'Super rice-7’' & $18.81^{* *}$ & $13.88^{* *}$ & $25.91^{* *}$ & $10.64^{* *}$ & $49.33^{* *}$ & $-25.32^{* *}$ & $-26.24^{* *}$ & $-28.69^{* *}$ \\
\hline \multicolumn{9}{|c|}{ 'PUSA 6A' } \\
\hline 'Super rice-8' & $10.96^{* *}$ & $9.76^{*}$ & $19.50^{* *}$ & 5.02 & $15.04^{* *}$ & $-42.47^{* *}$ & $-43.73^{* *}$ & $-45.59^{* *}$ \\
\hline 'R1099-2569-1-1' & -2.05 & $-7.75^{*}$ & $13.66^{* *}$ & -0.11 & $7.71^{* *}$ & $-46.14^{* *}$ & $-45.68^{* *}$ & $-47.49^{* *}$ \\
\hline 'R1557-1306-1-568-1' & $10.85^{* *}$ & 7.36 & $16.90^{* *}$ & 2.73 & $-73.27^{* *}$ & $-86.63^{* *}$ & $-87.40^{* *}$ & $-87.82^{* *}$ \\
\hline 'SR-6-SW-8-1' & 0.15 & -5.32 & $15.73^{* *}$ & 1.70 & $30.47^{* *}$ & $-34.75^{* *}$ & $-39.00^{* *}$ & $-41.02^{* *}$ \\
\hline 'Jitpiti' & $12.33^{* *}$ & $11.63^{* *}$ & $23.08^{* *}$ & $8.16^{*}$ & $34.65^{* *}$ & $-32.66^{* *}$ & $-34.29^{* *}$ & $-36.47^{* *}$ \\
\hline 'Super rice-7' & $8.82^{*}$ & $7.99^{*}$ & $19.40^{* *}$ & 4.92 & $65.56^{* *}$ & $-17.21^{* *}$ & $-18.23^{* *}$ & $-20.95^{* *}$ \\
\hline
\end{tabular}


Tab. 3. Percent relative heterosis, heterobeltiosis and standard heterosis for different characters in rice (Continuous)

\begin{tabular}{|c|c|c|c|c|c|c|c|c|}
\hline & \multicolumn{4}{|c|}{ Spikelet fertility (\%) } & \multicolumn{4}{|c|}{1000 grain weight $(\mathrm{g})$} \\
\hline & MP & $\mathrm{BP}$ & 'Mahamaya' & 'Indirasona' & MP & $\mathrm{BP}$ & 'Mahamaya' & 'Indirasona' \\
\hline \multicolumn{9}{|c|}{ 'APMS 6A' } \\
\hline 'Super rice-8' & $-6.27^{* *}$ & $-10.04^{* *}$ & $-10.93^{* *}$ & $-12.53^{* *}$ & 5.93 & -2.96 & $-23.40^{* *}$ & -6.48 \\
\hline 'R1099-2569-1-1' & $-22.42^{* *}$ & $-25.80^{* *}$ & $-25.99^{* *}$ & $-27.33^{* *}$ & 3.68 & -0.37 & $-29.02^{* *}$ & $-13.33^{* *}$ \\
\hline 'R1557-1306-1-568-1' & $-96.01^{* *}$ & $-96.03^{* *}$ & $-96.35^{* *}$ & $-96.42^{* *}$ & $-21.40^{* *}$ & $-27.45^{* *}$ & $-43.68^{* *}$ & $-31.24^{* *}$ \\
\hline 'SR-6-SW-8-1' & $-98.39^{* *}$ & $-98.40^{* *}$ & $-98.53^{* *}$ & $-98.56^{* *}$ & $-6.99^{*}$ & $-13.43^{* *}$ & $-34.01^{* *}$ & $-19.43^{* *}$ \\
\hline 'Jitpiti' & $-13.78^{* *}$ & $-16.22^{* *}$ & $-19.14^{* *}$ & $-20.60^{* *}$ & $-11.67^{* *}$ & $-18.52^{* *}$ & $-36.66^{* *}$ & $-22.67^{* *}$ \\
\hline 'Super rice-7' & $-39.39^{* *}$ & $-40.84^{* *}$ & $-43.42^{* *}$ & $-44.44^{* *}$ & -4.20 & $-14.12^{* *}$ & $-28.86^{* *}$ & $-13.14^{* *}$ \\
\hline \multicolumn{9}{|c|}{ 'CRMS 32A' } \\
\hline 'Super rice-8' & $-17.91^{* *}$ & $-20.25^{* *}$ & $-16.28^{* *}$ & $-17.78^{* *}$ & $10.00^{* *}$ & -2.17 & $-22.78^{* *}$ & -5.71 \\
\hline 'R1099-2569-1-1' & $-14.06^{* *}$ & $-16.21^{* *}$ & $-12.03^{* *}$ & $-13.62^{* *}$ & $11.67^{* *}$ & 4.01 & $-25.90^{* *}$ & $-9.52^{* *}$ \\
\hline 'R1557-1306-1-568-1' & $-98.92^{* *}$ & $-98.99^{* *}$ & $-98.94^{* *}$ & $-98.96^{* *}$ & -1.30 & $-11.58^{* *}$ & $-31.36^{* *}$ & $-16.19^{* *}$ \\
\hline 'SR-6-SW-8-1' & $-48.75^{* *}$ & $-52.03^{* *}$ & $-49.64^{* *}$ & $-50.55^{* *}$ & $-10.27^{* *}$ & $-18.95^{* *}$ & $-38.22^{* *}$ & $-24.57^{* *}$ \\
\hline 'Jitpiti' & $-29.47^{* *}$ & $-32.32^{* *}$ & $-28.95^{* *}$ & $-30.23^{* *}$ & $10.95^{* *}$ & -0.66 & $-22.78^{* *}$ & -5.71 \\
\hline 'Super rice-7’' & $-30.46^{* *}$ & $-33.55^{* *}$ & $-30.24^{* *}$ & $-31.50^{* *}$ & 1.62 & $-11.49^{* *}$ & $-26.68^{* *}$ & $-10.48^{* *}$ \\
\hline \multicolumn{9}{|c|}{ 'PUSA 6A' } \\
\hline 'Super rice- 8 ' & $-42.83^{* *}$ & $-46.77^{* *}$ & $-47.30^{* *}$ & $-48.24^{* *}$ & $13.91^{* *}$ & 3.56 & $-18.25^{* *}$ & -0.19 \\
\hline 'R1099-2569-1-1' & $-42.40^{* *}$ & $-46.55^{* *}$ & $-46.69^{* *}$ & $-47.65^{* *}$ & 7.04 & 2.04 & $-27.30^{* *}$ & $-11.24^{* *}$ \\
\hline 'R1557-1306-1-568-1' & $-88.04^{* *}$ & $-88.47^{* *}$ & $-89.39^{* *}$ & $-89.58^{* *}$ & 0.48 & $-7.96^{*}$ & $-28.55^{* *}$ & $-12.76^{* *}$ \\
\hline 'SR-6-SW-8-1' & $-34.19^{* *}$ & $-36.41^{* *}$ & $-41.78^{* *}$ & $-42.82^{* *}$ & $7.25^{*}$ & -0.94 & $-24.49^{* *}$ & $-7.81^{*}$ \\
\hline 'Jitpiti' & $-32.58^{* *}$ & $-36.47^{* *}$ & $-38.68^{* *}$ & $-39.79^{* *}$ & $10.93^{* *}$ & 1.55 & $-21.06^{* *}$ & -3.62 \\
\hline 'Super rice-7' & $-10.67^{* *}$ & $-15.46^{* *}$ & $-19.14^{* *}$ & $-20.60^{* *}$ & 4.76 & $-6.78^{*}$ & $-22.78^{* *}$ & -5.71 \\
\hline & \multicolumn{4}{|c|}{ Biological yield (g) } & \multicolumn{4}{|c|}{ Grain yield $(\mathrm{g})$} \\
\hline & $\mathrm{MP}$ & $\mathrm{BP}$ & 'Mahamaya' & 'Indirasona' & MP & $\mathrm{BP}$ & 'Mahamaya' & 'Indirasona' \\
\hline \multicolumn{9}{|c|}{ 'APMS 6A' } \\
\hline 'Super rice-8' & $45.76^{* *}$ & $23.06^{* *}$ & $14.81^{* *}$ & 0.13 & $82.37^{* *}$ & $68.37^{* *}$ & -2.69 & 6.16 \\
\hline 'R1099-2569-1-1' & $45.33^{* *}$ & $13.38^{* *}$ & $29.99^{* *}$ & $13.38^{* *}$ & $20.45^{* *}$ & 2.98 & $-29.03^{* *}$ & $-22.58^{* *}$ \\
\hline 'R1557-1306-1-568-1' & $34.89^{* *}$ & $33.18^{* *}$ & $-12.22^{* *}$ & $-23.44^{* *}$ & $-31.62^{* *}$ & $-46.15^{* *}$ & $-73.66^{* *}$ & $-71.26^{* *}$ \\
\hline 'SR-6-SW-8-1' & $15.30^{* *}$ & -0.03 & $-12.53^{* *}$ & $-23.71^{* *}$ & $-18.58^{* *}$ & $-27.16^{* *}$ & $-54.84^{* *}$ & $-50.73^{* *}$ \\
\hline 'Jitpiti' & $58.11^{* *}$ & $36.58^{* *}$ & $20.58^{* *}$ & 5.17 & $79.17^{* *}$ & $67.70^{* *}$ & -5.91 & 2.64 \\
\hline 'Super rice-7' & $-35.90^{* *}$ & $-42.05^{* *}$ & $-53.94^{* *}$ & $-59.83^{* *}$ & $-18.40^{* *}$ & $-27.06^{* *}$ & $-54.70^{* *}$ & $-50.59^{* *}$ \\
\hline \multicolumn{9}{|c|}{ 'CRMS 32A' } \\
\hline 'Super rice-8' & $53.20^{* *}$ & $46.17^{* *}$ & $36.37^{* *}$ & $18.94^{* *}$ & $59.34^{* *}$ & $42.65^{* *}$ & 4.30 & $13.78^{* *}$ \\
\hline 'R1099-2569-1-1' & $38.92^{* *}$ & $20.79^{* *}$ & $38.50^{* *}$ & $20.79^{* *}$ & $62.01^{* *}$ & $57.35^{* *}$ & $15.05^{* *}$ & $25.51^{* *}$ \\
\hline 'R1557-1306-1-568-1' & $53.23^{* *}$ & $36.20^{* *}$ & $15.41^{* *}$ & 0.66 & $-57.52^{* *}$ & $-70.59^{* *}$ & $-78.49^{* *}$ & $-76.54^{* *}$ \\
\hline 'SR-6-SW-8-1' & $-8.65^{*}$ & $-10.09^{*}$ & $-21.34^{* *}$ & $-31.39^{* *}$ & $-33.95^{* *}$ & $-38.97^{* *}$ & $-55.38^{* *}$ & $-51.32^{* *}$ \\
\hline 'Jitpiti' & $55.53^{* *}$ & $52.40^{* *}$ & $34.55^{* *}$ & $17.35^{* *}$ & $28.56^{* *}$ & $13.60^{* *}$ & $-16.94^{* *}$ & $-9.38^{*}$ \\
\hline 'Super rice- 7 ' & -1.60 & -4.66 & $-19.21^{* *}$ & $-29.54^{* *}$ & 6.36 & -1.65 & $-28.09^{* *}$ & $-21.55^{* *}$ \\
\hline \multicolumn{9}{|c|}{ 'PUSA 6A' } \\
\hline 'Super rice-8' & $-27.88^{* *}$ & $-41.81^{* *}$ & $-45.71^{* *}$ & $-52.65^{* *}$ & -0.27 & $-13.95^{*}$ & $-50.27^{* *}$ & $-45.75^{* *}$ \\
\hline 'R1099-2569-1-1' & $-49.82^{* *}$ & $-62.38^{* *}$ & $-56.87^{* *}$ & $-62.38^{* *}$ & $-35.01^{* *}$ & $-47.73^{* *}$ & $-63.98^{* *}$ & $-60.70^{* *}$ \\
\hline 'R1557-1306-1-568-1' & $64.24^{* *}$ & $53.46^{* *}$ & 1.14 & $-11.79^{* *}$ & -4.85 & $-20.51^{*}$ & $-66.67^{* *}$ & $-63.64^{* *}$ \\
\hline 'SR-6-SW-8-1' & $29.57^{* *}$ & 7.18 & -6.23 & $-18.21^{* *}$ & $-26.55^{* *}$ & $-38.43^{* *}$ & $-61.83^{* *}$ & $-58.36^{* *}$ \\
\hline 'Jitpiti' & $-28.21^{* *}$ & $-40.83^{* *}$ & $-47.76^{* *}$ & $-54.44^{* *}$ & $24.76^{* *}$ & 9.01 & $-38.84^{* *}$ & $-33.28^{* *}$ \\
\hline 'Super rice-7’ & 2.74 & $-11.63^{* *}$ & $-29.76^{* *}$ & $-38.74^{* *}$ & $62.79^{* *}$ & $36.36^{* *}$ & $-15.32^{* *}$ & $-7.62^{*}$ \\
\hline
\end{tabular}


96

SCA effects of hybrids (Tab. 4) revealed that highest SCA effect shown by 'CRMS 32A'/'R1099-2569-1-1' for grain yield and other contributing characters like pollen fertility, spikelet fertility and biological yield. 'APMS 6A'/'Super rice-8' and 'APMS $6 \mathrm{~A}^{\prime} /$ 'Jitpiti' is specific combiner for characters like plant height, pollen fertility, spikelet fertility, biological yield and grain yield. Similar results have been reported by Kumar et al. (2006), Jelodar and Bagheri (2010). Specific combining ability is the index to determine the usefulness of particular cross combination in the exploitation of heterosis.

\section{Heterosis}

For exploitation of hybrid rice breeding it is important to identify the potential restorers and maintainers from the existing rice germplasm. Percent heterosis for grain yield and yield related traits was calculated over mid parent, better parent and check varieties. The magnitude of heterosis varied from trait to trait, and cross to cross and none of the cross combination recorded significant heterosis for all the traits simultaneously (Tab. 3). These results are in agreement with the findings of Bagheri and Jelodar (2010), Rashid et al. (2007), Singh et al. (2005), Hussain and Sanghera (2012). Heterosis for grain yield along with its components is very important consideration in heterosis breeding. Yield is a complex character and ultimate aim of plant breeding. Highly significant and maximum positive heterosis was observed for grain yield in the hybrids viz.,'CRMS 32A'/'Super rice-8', 'APMS 6A'/'Super rice8', 'APMS 6A'/'Jitpiti' and 'CRMS 32A'/'R 1099-25691-1'. A high degree of heterobeltiosis was observed for grain yield in the hybrids viz., 'CRMS 32A'/'Super rice-8', 'CRMS 32A'/'R 1099-2569-1-1', 'APMS 6A'/'Super rice8 ' and 'APMS $6 \mathrm{~A}^{\prime} /$ 'Jitpiti'. A high degree of standard heterosis was observed for grain yield in the hybrid 'CRMS $32 A^{\prime} /$ 'R 1099-2569-1-1'. A high degree of relative heterosis, heterobeltiosis and standard heterosis was observed for the hybrid of 'CRMS 32A'/'R 1099-2569-1-1' (Tab. 3). Similar findings have been reported by Patnaik et al. (1990), Ali and Khan (1995), Parihar and Pathak (2008). The hybrids showing positive and significant SCA effects and values of relative heterosis, heterobeltiosis and standard heterosis will be further tested in observational yield/ multi-location yield trials to exploit its heterotic potential and fertility.

\section{References}

Ali SS, Khan MG (1995). Studies on heterosis and combining ability in rice. Pak J Sci Industr Rese 83:5-6.

Anand G, Amrithadevarathinum A, Edwin RJ (1999). Analysis in rice (Oryza sativa L.) under cold condition. Crop Res 15:91-93.

Anonymous (2011). Directorate of Economics and Statistics, Department of Agriculture and Cooperation, Ministry of Agriculture, Government of India, New Delhi.
Faiz FA, Sabar M, Awan TH, Ijaz M, Manzoor Z (2006). Heterosis and combining ability analysis in basmati rice hybrids. J Animal Plant Sci 16:1-2.

Fehr WR (1987). Heterosis, 115 p. In: Principles of cultivar development: Theory and Techniques (Vol.1). Macmillan Publishing Company. New York.

Hossain M (1988). Nature and Impact of the Green Revolution in Bangladesh. Research Report No. 67. Washington, D.C (USA). International Food Policy Research Institute.

Hussain W, Sanghera GS (2012). Exploitation of heterosis in rice (Oryza sativa L.) using CMS system under temperate conditions. Electr J Plant Breed 3(1):695-700.

Jayasudha S, Sharma D (2009). Combining ability and gene action analysis for yield and its components in rice (Oryza sativa L.). J Rice Res 2:105-110.

Jelodar NB, Bagheri N (2010). Heterosis and combining ability analysis for yield and related-yield traits in hybrid rice. Int J Biol 2(2):222-227.

Kempthorne O (1957). An introduction to genetic studies. John Willey and Sons Inc., New York, USA.

Kumar A, Singh NK, Sharama VK (2006). Combining ability analysis for identifying elite parents for heterotic rice hybrids. Oryza 43:82-86.

Khush GS (1997). Breaking the yield barrier of rice. Geo J 35:329-332.

Lin SC, Yuan LP (1980). Hybrid rice breeding in China, 35-51 p. In: Innovative approaches to rice breeding. Manila (Philippines). International Rice Research Institute.

Nadarajan N, Gunasekaran M (2005). Quantitative genetics and biometrical techniques in plant breeding. Kalyani publishers, New Delhi, 57-60 p.

Parihar A, Pathak AR (2008). Heterosis for various quantitative traits in rice. Oryza 45(3):181-187.

Patnaik RN, Pandey K, Rathor SN, Jachuck PJ (1990). Heterosis in rice hybrids. Euphytica 49(3):243-247.

Rahimi R, Rabiei B, Samizadeh H, Kafi Ghasemi A (2010). Combining ability and heterosis in rice cultivars. J Agric Sci Technol 12:223-231.

Rai M (2009). Hybrid culture in sustainable agriculture, 3-17 p. In: Proc of the $9^{\text {th }}$ Agricultural Science Congress, held at SKUAST-K, Shalimar, Srinagar, 22-24 June.

Rashid M, Cheema AA, Ashraf M (2007). Line x Tester analysis in basmati rice. Pak J Bot 39(6):2035-2042.

Saleem MY, Mirza JI, Haq MA (2010). Combining ability analysis for yield and related traits in basmati rice (Oryza sativa L.). Pak J Bot 42(1):627-637.

Salgotra RK, Gupta BB, Praveen S (2009). Combining ability studies for yield and yield components in basmati rice. Oryza 46(1):12-16.

Sanghera GS, Wani SH (2008). Innovative approaches to enhance genetic potential of rice (Oryza sativa L.) for higher productivity under temperate conditions of Kashmir. The J 


\section{Plant Sci Res 24:99-113.}

Sanghera GS, Hussain W (2012a). Heterosis and combining ability estimates using line $\mathrm{x}$ tester analysis to develop rice hybrids for temperate conditions. Not Sci Biol 4(3):131142.

Sanghera GS, Hussain W (2012b). Study on heterosis in relation to combining ability per se performance in temperate rice (Oryza sativa L.). Elixir Appl Bot 49:10048-10054.

Sarkar U, Biswas PS, Prasad B, Khaleque Mian MA (2002). Heterosis and genetic analysis in rice hybrid. Pak J Biol Sci 5(1):1-5.

Singh RV, Maurya DM, Dwivedi JL, Verma OP (2005). Combining ability studies on yield and its components using CMS lines in rice (Oryza sativa L.). Oryza 42:306-309.

Swaminathan MS (2006). An evergreen revolution. Crop Sci 46:2293-2303.
Swaminathan MS (1998). Gender dimensions in biodiversity management. New Delhi: Konark Publishers Pvt Ltd.

Tiwari DK, Pandey P, Giri SP, Dwivedi JL (2011). Heterosis studies for yield and its components in rice hybrids using CMS system. Asian J Plant Sci 10:29-42.

Vanaja T, Luckins C, Babu V, Radhakrishnan V, Pushkaran K (2003). Combining ability analysis for yield and yield components in rice varieties of diverse origin. Tropical Agric 41:7-15.

Virmani SS (1996). Hybrid rice. Adv Agron 57:328-462.

Virmani SS, Mao CX, Hardy B (2003). Hybrid rice for food security, poverty alleviation, and environmental protection, 407 p. In: Proc of the $4^{\text {th }}$ International Sympo on Hybrid Rice, held at Hanoi, Vietnam, 14-17 May. 